\title{
PROJETO DE ESTABILIZADORES MULTI-BANDA UTILIZANDO ALOCAÇÃ̃O DE POLOS VIA MÉTODO DE NEWTON-RAPHSON
}

\author{
Wesley Peres. Francisco C. R. Coelho. \\ Programa de Pós-Graduação em Engenharia Elétrica \\ (PPGEL/UFSJ/CEFET-MG), Departamento de Engenharia Elétrica \\ (DEPEL), Universidade Federal de São João del-Rei (UFSJ), São \\ João-del Rei, MG, Brasil \\ (e-mails: wesley.peres@ufsj.edu.br; franciscocoelho@ufsj.edu.br).
}

\begin{abstract}
This paper presents an approach for multi-band power system stabilizer tuning based on the pole placement technique solved by the Newton-Raphson method. The main objective is to move unstable and undamped eigenvalues to satisfactory locations in the complex plane defined by a minimum damping ratio in closed loop operation. Initial results for the well known Single Machine Infinite Bus system show that the benefits of the proposed approach.
\end{abstract}

Resumo: O presente trabalho apresenta uma metodologia para o ajuste de estabilizadores de sistemas de potência multi-banda baseado na técnica de alocação de polos via método de Newton-Raphson. O objetivo principal é mover os autovalores instáveis e fracamente amortecidos para regiões satisfatórias do plano complexo definidas por um coeficiente mínimo de amortecimento em malha fechada. Resultados iniciais obtidos para um Sistema Máquina Barra Infinita indicam os benefícios da metodologia proposta.

Keywords: Multi-Band power system stabilizers; Pole placement; Power system control; Power system and stability; Newton-Raphson.

Palavras-chaves: Estabilizadores de sistemas de potência multi-banda; Alocação de polos; Controle de sistemas de potência; Estabilidade de sistemas de potência; Método de Newton-Raphson.

\section{INTRODUÇÃO}

A análise da estabilidade a pequenas perturbações em sistemas elétricos de potência (SEP) corresponde ao estudo das oscilações eletromecânicas nos geradores que ocorrem após um desbalanço de torques elétrico e mecânico (devido a pequenas variações de carga ou geração). Tais oscilações de baixa frequência, conforme (Sauer \& Pai, 1998), podem ser locais (envolvendo um grupo de geradores e o resto do sistema, na faixa de 1-3 Hz) ou interarea (envolvendo dois ou mais grupos de geradores em diferentes áreas, na faixa de 0,1 a $1 \mathrm{~Hz}$ ). Existem ainda, de acordo com (Grondin et al., 2003), os modos de oscilação globais $(0,01 \mathrm{a} 0,1 \mathrm{~Hz})$, observados em sistemas isolados, fora do escopo desse artigo.

Os modos locais de oscilação são, desde a década de setenta, amortecidos através dos Estabilizadores de Sistemas de Potência (ESP) que fazem o controle do sistema de excitação dos geradores síncronos no regime transitório. Por outro lado, como reportado em (Ferraz et al., 2007), o amortecimento dos modos interarea, por serem de natureza global, precisa de uma estratégia de projeto mais elaborada, como a aplicação de técnicas de ajuste coordenado (simultâneo) dos diversos controladores do sistema.

Os ESP convencionais, tratados nos parágrafos anteriores, são largamente aplicados na indústria e baseados na potência acelerante, sintetizada nos terminais das máquinas (sinais locais). Como apresentado em (Peres et al., 2018), estes são controladores com estágios de ganho, compensação de fase e filtros, ajustados por técnicas clássicas de controle, controle robusto e técnicas de otimização.

Visando a obtenção de um estabilizador com a capacidade de prover amortecimento ao sistema em uma ampla faixa de frequência, em 2000 (Grondin et al., 2003) propôs o ESP Multi-Banda (ESP-MB), padronizado em (IEEE, 2006) como PSS4B. Este é baseado em uma estrutura de controle flexível e consiste de três bandas que correspondem a três faixas de frequência ou modos de oscilação: (i) baixa (modos globais), (ii) intermediária (modos interárea) e (iii) alta (modos locais). Cada banda contém dois ramos com blocos de ganho, compensação de fase e blocos híbridos.

Conforme (Rimorov et al., 2015), a falta de metodologias de ajuste dos ESP-MB é a maior barreira para a disseminação desses controladores nos sistemas de energia. Nesse contexto, o desenvolvimento de ferramentas de ajuste dos ESP-MB é um campo atual e fértil de pesquisa.

Uma metodologia, bastante utilizada na prática e que será revisada na próxima seção, foi proposta por (Grondin et al., 2003) e é baseada no uso de filtros passa-banda, para os quais é feito o ajuste de frequências centrais e ganhos nessas frequências. Ferramentas de otimização também foram utilizadas e por questões de espaço, tal revisão não será detalhada aqui, sendo o leitor referenciado à (Peres et al., 
2018) para maiores detalhes. As metodologias de otimização propostas são baseadas em metaheuristicas (Enxame de Partículas, Computação Evolutiva, Busca Harmônica, Evolução Diferencial, Ecolocalização de Morcegos e Busca Gravitacional) ou métodos analíticos de otimização.

Este trabalho apresenta uma ferramenta de ajuste de ganhos do ESP-MB baseada na técnica de alocação de polos via método de Newton-Raphson. Uma ferramenta similar foi empregada em (Ferraz et al., 2007) para o ajuste de estabilizadores convencionais. O objetivo é mover os autovalores instáveis e/ou fracamente amortecidos para regiões satisfatórias do plano complexo definidas por um coeficiente mínimo de amortecimento em malha fechada. Uma análise inicial para um Sistema Máquina Barra Infinita (SMBINF) indica que resultados promissores podem ser obtidos.

\section{O ESTABILIZADOR MULTI-BANDA (ESP-MB)}

A modelagem completa do ESP-MB pode ser obtida em (Kamwa et al., 2005). O presente trabalho é focado em uma variação do modelo simplificado do ESP-MB proposto em (Grondin et al., 2003) e apresentado na Fig. 1.

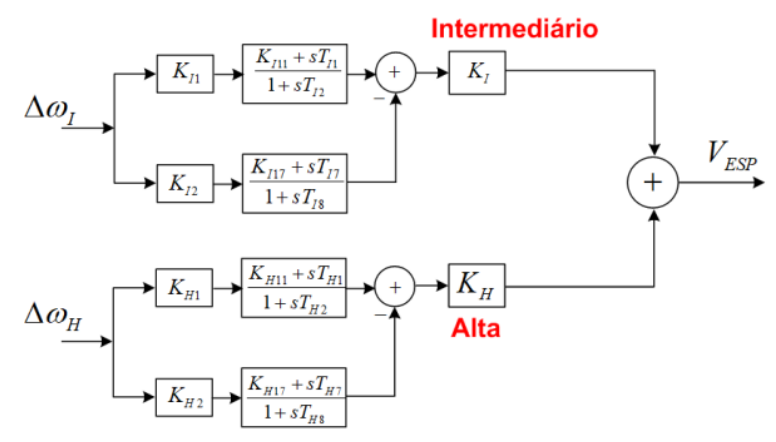

Fig. 1 Representação do modelo simplificado do ESP-MB.

A Fig. 2 ilustra, didaticamente, a representação do modelo simplificado do ESP-MB usado nesse trabalho. Nesse modelo, cada banda é analisada como um filtro passa banda e existem quatro parâmetros de ajuste:

i. Frequências Centrais das bandas: intermediária $\left(\mathrm{F}_{\mathrm{I}}\right)$ e alta $\left(\mathrm{F}_{\mathrm{H}}\right)$. $\quad$ É importante mencionar que essa metodologia simplificada permite obter filtros passa banda com frequências centrais bem separadas, que é o propósito do ESP-MB;

ii. Ganhos nas frequências centrais para as duas bandas: intermediária $\left(\mathrm{K}_{\mathrm{I}}\right)$ e alta $\left(\mathrm{K}_{\mathrm{H}}\right)$.

Observa-se que, neste trabalho, trabalha-se somente com as bandas intermediária e alta. Adicionalmente, os sinais de entrada do modelo simplificado $\left(\Delta \omega_{I}\right.$ e $\left.\Delta \omega_{H}\right)$ são obtidos a partir de transdutores conforme mostrado em (IEEE, 2006). Nesse caso, a entrada dos transdutores é a velocidade terminal da máquina $(\Delta \omega)$.
Uma vez que as frequências centrais e os respectivos ganhos são conhecidos, é possível calcular as constantes de tempo do estabilizador ilustrado na Fig. 1, conforme apresentado nas equações (1)-(5). Conforme (Kamwa et al., 2005), a constante $R$, que controla a largura da banda, é ajustada em 1,2. Essas equações estão associadas à banda de frequência intermediária e podem ser estendidas para a banda alta.

$$
\begin{gathered}
T_{I 2}=T_{I 7}=\frac{1}{2 \cdot \pi \cdot F_{I} \cdot \sqrt{R}} \\
T_{I 1}=T_{I 2} / R \\
T_{I 8}=T_{I 7} \cdot R \\
K_{I 1}=K_{I 2}=\frac{R^{2}+R}{R^{2}-2 R+1} \\
K_{I 11}=K_{I 17}=1
\end{gathered}
$$

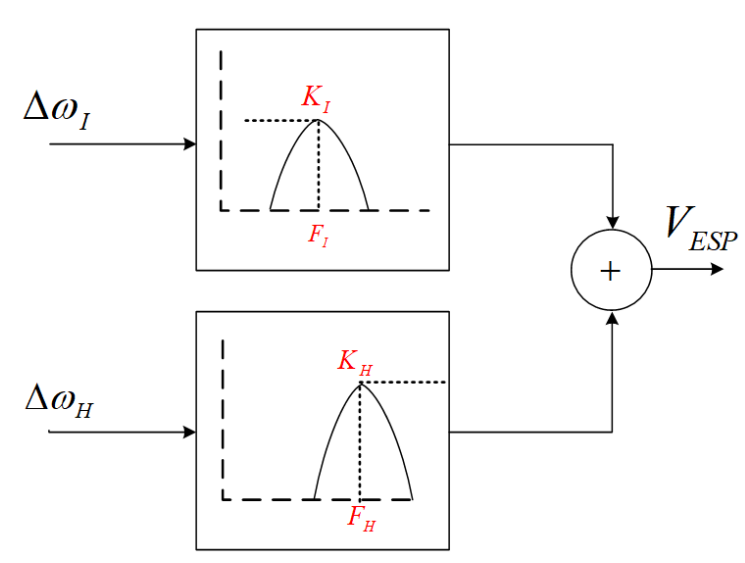

Fig. 2 Representação do modelo simplificado do ESP-MB.

Adicionalmente se pode utilizar, a priori, as frequências centrais típicas definidas em (IEEE, 2006) para a banda intermediária e alta: $\mathrm{F}_{\mathrm{I}}=0,7 \mathrm{~Hz}$ e $\mathrm{F}_{\mathrm{H}}=8,0 \mathrm{~Hz}$. Com isso, para cada estabilizador, existem somente dois ganhos a serem ajustados: $\mathrm{K}_{\mathrm{I}}$ e $\mathrm{K}_{\mathrm{H}}$. Esse procedimento foi adotado nesse artigo, apresentando bons resultados.

\section{REPRESENTAÇÃO DO SISTEMA}

A modelagem adotada para o sistema segue a referência (Sauer and Pai, 1998): (i) o gerador é representado por um modelo diferencial de $3^{\mathrm{a}}$ ordem e (ii) o gerador é equipado por um regulador automático de tensão estático (de primeira ordem). O SEP em malha aberta é representado por sua função de transferência $S E P(s)=\Delta \omega(s) / \Delta V_{R E F}(s)$, sendo $\Delta \omega(s)$ a velocidade terminal em pu e $\Delta V_{R E F}(s)$ a tensão de referência do regulador de tensão. Os autovalores em malha aberta são as raízes do denominador dessa função.

A Fig. 3 ilustra o sistema realimentado, em que a função de transferência do ESP-MB $P S S 4 B(s)$ é destacada. A partir da realimentação é possível calcular os autovalores em malha fechada. 


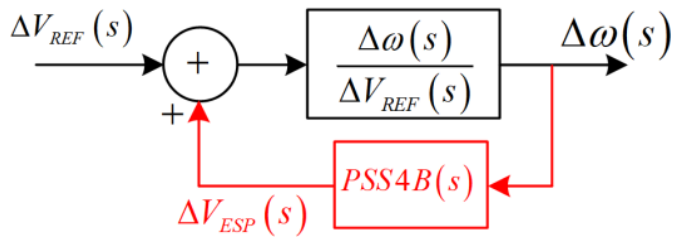

Fig. 3 Sistema de potência realimentado.

\section{METODOLOGIA PROPOSTA}

Nessa seção é apresentada a metodologia proposta, incluindo a sua formulação matemática e o seu algoritmo de solução.

\subsection{Variação de Polos para uma Variação de Ganho}

Considere um polo complexo e um ESP-MB representado pelos seus ganhos $\mathrm{K}_{\mathrm{I}}$ e $\mathrm{K}_{\mathrm{H}}$. A posição inicial do polo é apresentada na equação (6) (somente a parte de frequência positiva é apresentada).

$$
\lambda_{0}=\sigma_{0}+j \omega_{0}
$$

Uma variação de ganhos $\Delta \mathrm{K}_{\mathrm{I}}$ e $\Delta \mathrm{K}_{\mathrm{H}}$ causa um deslocamento de polo $\Delta \lambda$ dado pela equação (7).

$$
\Delta \lambda=\left[\frac{\partial \lambda}{\partial K_{I}}\right] \cdot\left[\Delta K_{I}\right]+\left[\frac{\partial \lambda}{\partial K_{H}}\right] \cdot\left[\Delta K_{H}\right]
$$

Sendo $\Delta \lambda=\lambda-\lambda_{0}$, tem-se a equação (8).

$$
\lambda=\lambda_{0}+\left[\frac{\partial \lambda}{\partial K_{I}}\right] \cdot\left[\Delta K_{I}\right]+\left[\frac{\partial \lambda}{\partial K_{H}}\right] \cdot\left[\Delta K_{H}\right]
$$

em que as derivadas parciais representam uma sensibilidade dos polos às variações nos ganhos. Os valores das sensibilidades podem ser obtidos numericamente.

A equação (8) pode ser reescrita considerando as suas partes real e imaginária, conforme apresentado na equação (9).

$$
\begin{gathered}
\sigma=\sigma_{0}+\left[\frac{\partial \sigma}{\partial K_{I}}\right] \cdot\left[\Delta K_{I}\right]+\left[\frac{\partial \sigma}{\partial K_{H}}\right] \cdot\left[\Delta K_{H}\right] \\
\omega=\omega_{0}+\left[\frac{\partial \omega}{\partial K_{I}}\right] \cdot\left[\Delta K_{I}\right]+\left[\frac{\partial \omega}{\partial K_{H}}\right] \cdot\left[\Delta K_{H}\right]
\end{gathered}
$$

Fazendo um equacionamento adequado, a partir das expressões anteriores, é possível calcular os valores dos ganhos para se alocar o polo em um valor desejado.

\subsection{Coeficiente de Amortecimento}

O coeficiente de amortecimento de um autovalor é definido pela equação (10).

$$
\xi=-\frac{\sigma}{\sqrt{\sigma^{2}+\omega^{2}}}
$$

Após algumas manipulações, pode-se apresentar a equação (10) conforme a equação (11).

$$
\sigma+\omega \cdot \frac{\xi}{\sqrt{1-\xi^{2}}}=0
$$

\subsection{Formulação Matricial}

As equações (9) e (11) podem ser reescritas matricialmente conforme a equação (12).

$$
\left[\begin{array}{cccc}
1 & 0 & -\frac{\partial \sigma}{\partial K_{I}} & -\frac{\partial \sigma}{\partial K_{H}} \\
0 & 1 & -\frac{\partial \omega}{\partial K_{I}} & -\frac{\partial \omega}{\partial K_{H}} \\
1 & \frac{\xi}{\sqrt{1-\xi^{2}}} & 0 & 0
\end{array}\right] \cdot\left[\begin{array}{c}
\sigma \\
\omega \\
\Delta K_{I} \\
\Delta K_{H}
\end{array}\right]=\left[\begin{array}{c}
\sigma_{0} \\
\omega_{0} \\
0
\end{array}\right]
$$

Considere agora a existência de dois pares de polos complexos $\lambda_{1}$ e $\lambda_{2}$ (somente a parte superior do plano é considerada) e um estabilizador ESP-MB. Pode-se calcular os incrementos de ganho $\Delta \mathrm{K}_{\mathrm{I}}$ e $\Delta \mathrm{K}_{\mathrm{H}}$ necessários para se alocar os polos em um ponto cujos amortecimentos desejados sejam $\xi_{\mathrm{d} 1}$ e $\xi_{\mathrm{d} 2}$. O sistema de equaçõos é apresentado em (13).

$$
\left[\begin{array}{ccccrr}
1 & 0 & 0 & 0 & -\frac{\partial \sigma_{1}}{\partial K_{I}} & -\frac{\partial \sigma_{1}}{\partial K_{H}} \\
0 & 1 & 0 & 0 & -\frac{\partial \sigma_{2}}{\partial K_{I}} & -\frac{\partial \sigma_{2}}{\partial K_{H}} \\
0 & 0 & 1 & 0 & -\frac{\partial \omega_{1}}{\partial K_{I}} & -\frac{\partial \omega_{1}}{\partial K_{H}} \\
0 & 0 & 0 & 1 & -\frac{\partial \omega_{2}}{\partial K_{I}} & -\frac{\partial \omega_{2}}{\partial K_{H}} \\
1 & 0 & \frac{\xi_{d 1}}{\sqrt{1-\xi_{d 1}^{2}}} & 0 & 0 & 0 \\
0 & 1 & 0 & \frac{\xi_{d 2}}{\sqrt{1-\xi_{d 2}^{2}}} & 0 & 0
\end{array}\right] \cdot\left[\begin{array}{c}
\sigma_{1} \\
\sigma_{2} \\
\omega_{1} \\
\omega_{2} \\
\Delta K_{I} \\
\Delta K_{H}
\end{array}\right]=\left[\begin{array}{c}
\sigma_{01} \\
\sigma_{02} \\
\omega_{01} \\
\omega_{02} \\
0 \\
0
\end{array}\right]
$$

O sistema de equações dado em (13) pode ser resolvido iterativamente de forma a calcular, partindo-se de uma condição inicial $\lambda_{01}$ e $\lambda_{02}$, o valor de ganhos $K_{I}$ e $K_{H}$ tal que os novos amortecimentos sejam $\xi_{\mathrm{d} 1}$ e $\xi_{\mathrm{d} 2}$. Matematicamente tem-se um problema de localização de raízes $\left(\mathrm{K}_{\mathrm{I}}\right.$ e $\left.\mathrm{K}_{\mathrm{H}}\right)$ resolvido pelo método de Newton-Raphson.

A estrutura do sistema de equações pode ser rearranjada convenientemente para diferentes números de polos e estabilizadores.

\subsection{Algoritmo Proposto}

O algoritmo proposto é apresentado na Fig. 4 e é descrito como segue:

Entrada: (i) matrizes em espaço de estados em malha aberta, (ii) valor das frequências centrais das bandas intermediária e alta, (iii) valor de amortecimento mínimo desejado $\xi_{\text {min }}$, (iv) número máximo de iterações do algoritmo, $(v)$ polos a serem alocados e (vi) ganhos iniciais $\mathrm{K}_{\mathrm{I} 0}$ e $\mathrm{K}_{\mathrm{H} 0}$. 
Saída: valores dos ganhos $\mathrm{K}_{\mathrm{I}}$ e $\mathrm{K}_{\mathrm{H}}$.



Fig. 4 Algoritmo proposto.

Passo 1: Iniciar o contador de iterações $(h=1)$.

Passo 2: Considerando os valores iniciais de ganhos $K_{I 0}$ e $\mathrm{K}_{\mathrm{H} 0}$ e as matrizes em malha aberta, fazer a realimentação e calcular os valores iniciais de cada polo a ser alocado $\lambda_{0}=\sigma_{0}+\mathrm{j} \omega_{0}$.

Passo 3: Calcular as sensibilidades (derivadas) de cada polo em relação aos ganhos das bandas intermediária e alta.

Passo 4: Considerando $\xi_{\mathrm{d} 1} \ldots \xi_{\mathrm{dn}}=\xi_{\min }$, resolver o sistema de equações dado em (13), sendo $n$ o número de polos a serem alocados. A solução do sistema de equações é realizada a partir do conceito de matriz pseudo-inversa e será discutido na seção seguinte.

Passo 5: Atualizar os ganhos conforme a equação (14). Calcular os novos autovalores $\lambda_{1} \ldots \lambda_{n}$ através de uma rotina de realimentação, para os quais é possível obter os coeficientes de amortecimento $\xi_{1} \ldots \xi_{n}$.

$$
\begin{gathered}
K_{I}=K_{I 0}+\Delta K_{I} \\
K_{H}=K_{H 0}+\Delta K_{H}
\end{gathered}
$$

Passo 6: Caso o amortecimento de todos os autovalores seja maior que o valor mínimo $\xi_{\text {min }}$, deve-se encerrar o processo iterativo e apresentar os valores de ganho. Isso equivale a dizer que todos os polos foram alocados em "uma região satisfatória de amortecimento" definida por um fator de amortecimento mínimo $\xi_{\min }$ (Fig. 5). Caso contrário, ir ao passo 7 .

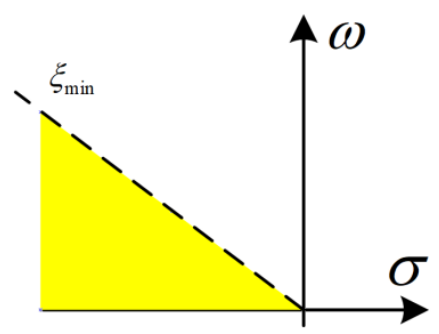

Fig. 5 Região satisfatória para alocação de polos.

Passo 7: Caso o algoritmo não tenha posicionado os polos na região satisfatória deve-se incrementar contador de iterações $(\mathrm{h}=\mathrm{h}+1)$. Caso o número de iterações seja maior que o máximo $\left(\mathrm{h}_{\max }\right)$, deve-se encerrar $\mathrm{o}$ processo iterativo (divergência). Caso contrário, fazer $\mathrm{K}_{\mathrm{I} 0}=\mathrm{K}_{\mathrm{I}}$ e $\mathrm{K}_{\mathrm{H} 0}=\mathrm{K}_{\mathrm{H}}$ e retornar ao Passo 3.

\subsection{Considerações sobre a Metodologia Proposta}

O algoritmo proposto é simples, entretanto algumas considerações devem ser feitas:

i. Na prática deseja-se satisfazer a um critério de amortecimento mínimo e não necessariamente alocar os polos exatamente sobre a reta definida por $\xi_{\text {min }}$ (Fig. 5). Isso difere a metodologia proposta nesse artigo da apresentada em (Ferraz, Martins and Taranto, 2007) (focada em estabilizadores convencionais);

ii. Os número de polos e "qual(is) o(s) polo(s)" a ser (em) alocado(s) devem ser previamente definidos. Em geral, utiliza-se aquele(s) menos amortecido(s) e/ou instáveis, que são de natureza eletromecânica.

iii. O sistema de equações dado em (13) não necessariamente apresenta o mesmo número de variáveis e equações. Nesse caso, faz-se uso do conceito de matriz pseudo-inversa (ou matriz inversa generalizada), que permite a solução de sistemas sobre ou subdeterminados (Ferraz, Martins and Taranto, 2007).

Considere o sistema linear de equações dado em (15). Se $p=q$, a matriz A é não singular e a solução de (15) é dada por (16), em que $[A]^{-1}=\left[\mathrm{A}_{p \times q}\right]^{-1}$ é a inversa de $[A]=$ $\left[\mathrm{A}_{p \times q}\right]$.

$$
\begin{gathered}
{\left[A_{p x q}\right]\left[x_{q x 1}\right]-\left[b_{p x 1}\right]=0} \\
{\left[x_{q x 1}\right]=\left[A_{p x q}\right]^{-1}\left[b_{p x 1}\right]}
\end{gathered}
$$

Se $p \neq q$, deve-se encontrar a matriz pseudo-inversa ou matriz inversa generalizada $\left[\mathrm{A}_{q \times p}\right]^{+}$para a solução de (16). 


$$
\left\{\begin{array}{l}
\text { se } q>p: A_{q x p}^{+}=A^{T} \cdot\left(A \cdot A^{T}\right)^{-1} \\
\text { se } q<p: A_{q x p}^{+}=\left(A^{T} \cdot A\right)^{-1} \cdot A^{T}
\end{array}\right.
$$

\section{ESTUDO DE CASO}

\subsection{Apresentação do Sistema}

A rede elétrica a ser utilizada nesse trabalho é o Sistema Máquina Barra Infinita (SMBINF) apresentada na Fig. 6 e adequada para o estudo de oscilações locais entre uma máquina e um sistema de grande porte. Os dados são dados em p.u. na base de 100 MVA.

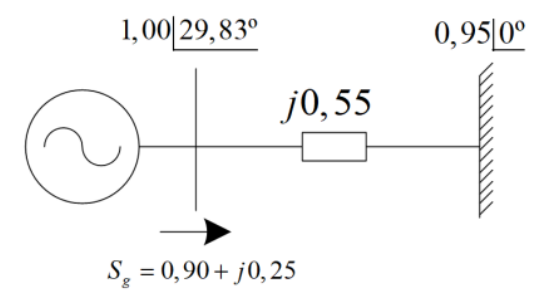

Fig. 6 Sistema Máquina Barra Infinita (SMBINF).

A máquina é representada por um modelo de terceira ordem cujos dados estão na Tabela 1. Os dados do sistema de excitação estático são apresentados na Tabela 2. Os modelos podem ser obtidos em (Sauer \& Pai, 1998).

Tabela 1. Dados da Máquina Síncrona.

\begin{tabular}{|c|c|}
\hline$H=3,0 \mathrm{seg}$ & $T_{d 0}^{\prime}=5,0 \mathrm{seg}$ \\
\hline$R_{s}=0,0 \mathrm{p} . u$. & $X_{q}=0,6 \mathrm{pu}$ \\
\hline$X_{d}=0,8958 \mathrm{p} . u$. & $X_{d}^{\prime}=0,1198 \mathrm{p} . u$. \\
\hline$D=0,0$ & $f_{e}=60 \mathrm{~Hz}$ \\
\hline
\end{tabular}

Tabela 2. Dados do Sistema de Excitação Estático.

\begin{tabular}{|l|l|}
$K_{a}=100,0 \mathrm{pu}$ & $T_{a}=0,05 \mathrm{seg}$ \\
\hline
\end{tabular}

\subsection{Sistema em Malha Aberta}

A Tabela 3 apresenta os polos (modos ou autovalores) do sistema em malha aberta. Observa-se a instabilidade do sistema devido a um modo oscilatório instável cujo amortecimento é negativo. Deve-se, portanto, proceder com a alocação e um ESP-MB e o seu ajuste através da metodologia proposta.

Tabela 3. Autovalores em Malha Aberta.

\begin{tabular}{|c|c|}
\hline Autovalores & Amortecimento (\%) \\
\hline $0,0052 \pm j 8,06$ & $-0,06$ \\
\hline$-10,22 \pm j 14,22$ & 58,36 \\
\hline
\end{tabular}

\subsection{Projeto do Estabilizador Multi-Banda}

Para o projeto do ESP-MB via a metodologia proposta, considera-se uma tolerância de $0,1 \%$ para o amortecimento mínimo $\xi_{\min }$ e um máximo de 25 iterações. Os seguintes parâmetros serão variados para efeitos de análise: (i) ganhos iniciais $\left(\mathrm{K}_{\mathrm{I} 0}\right.$ e $\left.\mathrm{K}_{\mathrm{H} 0}\right)$, (ii) número de polos monitorados (1 ou 2) e (iii) amortecimento mínimo desejado em malha fechada. A Tabela 4 e a Tabela 5 apresentam os fatores de amortecimento mínimo obtidos e os respectivos ganhos de banda intermediária e alta para diferentes valores desejados de amortecimento. Consideram-se, nesse caso, dois polos no processo de monitoramento, definidos previamente no início do processo iterativo (somente a parte superior do plano complexo é utilizada).

Tabela 4. Amortecimento Mínimo Alcançado considerando 2 polos monitorados no processo de alocação.

\begin{tabular}{|l|c|c|c|c|c|c|}
\hline \multirow{2}{*}[\mathrm{K}_{\mathrm{I}0},\mathrm{K}_{\mathrm{H}0}]{} & \multicolumn{6}{|c|}{ Mínimo Amortecimento Desejado $\left(\xi_{\min }\right)$} \\
\cline { 2 - 7 } & $5 \%$ & $10 \%$ & $15 \%$ & $20 \%$ & $25 \%$ & $30 \%$ \\
\hline$[5,5]$ & 7,73 & 11,24 & 15,42 & 20,72 & 24,94 & DIV \\
\hline$[5,10]$ & 4,92 & 10,06 & 15,76 & 20,19 & 25,00 & DIV \\
\hline$[5,50]$ & 5,00 & 10,00 & 14,95 & 20,00 & 25,00 & DIV \\
\hline
\end{tabular}

Tabela 5. Valores dos Ganhos Ajustados $\left[\mathbf{K}_{\mathbf{I}}, \mathbf{K}_{\mathbf{H}}\right]$ considerando 2 polos monitorados no processo de alocação.

\begin{tabular}{|c|c|c|c|c|c|c|}
\hline \multirow{2}{*}[\mathrm{K}_{\mathrm{I}0},\mathrm{K}_{\mathrm{H}0}]{} & \multicolumn{6}{|c|}{ Mínimo Amortecimento Desejado $\left(\xi_{\min }\right)$} \\
\cline { 2 - 7 } & $5 \%$ & $10 \%$ & $15 \%$ & $20 \%$ & $25 \%$ & $30 \%$ \\
\hline$[5,5]$ & 3,99 & 13,33 & 16,74 & 11,39 & 57,36 & \multirow{2}{*}{ DIV } \\
& 131,2 & 111,6 & 92,95 & 72,67 & 79,31 & \\
\hline$[5,10]$ & $-1,34$ & 1,80 & 5,97 & 8,95 & 15,00 & \multirow{2}{*}{ IV } \\
& 149,3 & 109,9 & 89,84 & 73,13 & 61,61 & \\
\hline$[5,50]$ & $-1,24$ & 2,04 & 5,16 & 8,78 & 14,99 & \multirow{2}{*}{ DIV } \\
& 149,8 & 117,7 & 92,69 & 74,34 & 61,61 & \\
\hline
\end{tabular}

Considerando diferentes condições iniciais, para um mesmo valor de $\xi_{\min }$, observa-se que os valores de amortecimento variam pouco, enquanto os ganhos da banda intermediária $\left(\mathrm{K}_{\mathrm{I}}\right)$ sofrem variações significativas. Adicionalmente nota-se o ganho $\mathrm{K}_{\mathrm{I}}$ negativo obtido em dois casos considerados. Para um $\xi_{\min }$ desejado de $30 \%$, o processo não alcançou convergência.

O tempo médio de simulação foi de 1,5 segundos em 3 iterações, em um computador Intel Core i5, $2.40 \mathrm{GHz}$ com 8 GB de RAM e sistema operacional Windows 10, 64 bits (usando a plataforma Matlab).

$\mathrm{Na}$ segunda simulação, considera-se um único polo monitorado no processo iterativo. $\mathrm{O}$ polo selecionado foi o instável em malha aberta $0,0052 \pm j 8,06$. Ressalta-se que esse polo não é alterado durante a solução e os resultados são apresentados na Tabela 6 e na Tabela 7. O número médio de iterações foi 4 em 1,5 segundos. Ressalta-se que a redução do número de polos monitorados implica na redução da dimensão das matrizes.

Tabela 6. Amortecimento Mínimo Alcançado considerando 1 polo monitorado no processo de alocação.

\begin{tabular}{|c|c|c|c|c|c|c|}
\hline \multirow{2}{*}[\mathrm{K}_{\mathrm{I}0},\mathrm{K}_{\mathrm{H}0}]{} & \multicolumn{5}{|c|}{ Mínimo Amortecimento Desejado $\left(\xi_{\min }\right)$} \\
\cline { 2 - 7 } & $5 \%$ & $10 \%$ & $15 \%$ & $20 \%$ & $25 \%$ & $30 \%$ \\
\hline$[5,5]$ & 4,95 & 9,92 & 15,00 & 19,96 & 24,97 & 21,80 \\
\hline$[5,10]$ & 5,01 & 10,00 & 14,91 & 19,92 & 24,95 & 21,83 \\
\hline$[5,50]$ & 5,68 & 10,10 & 15,00 & 20,00 & 24,91 & 22,03 \\
\hline
\end{tabular}


Tabela 7. Valores dos Ganhos Ajustados $\left[\mathbf{K}_{\mathbf{I}}, \mathbf{K}_{\mathbf{H}}\right]$ considerando 1 polo monitorado no processo de alocação.

\begin{tabular}{|l|c|c|c|c|c|c|}
\hline \multirow{2}{*}[\mathrm{K}_{\mathrm{I}0},\mathrm{K}_{\mathrm{H}0}]{} & \multicolumn{6}{|c|}{ Mínimo Amortecimento Desejado $\left(\xi_{\min }\right)$} \\
\cline { 2 - 7 } & $5 \%$ & $10 \%$ & $15 \%$ & $20 \%$ & $25 \%$ & $30 \%$ \\
\hline$[5,5]$ & 3,60 & 5,68 & 9,38 & 13,19 & 17,63 & 22,60 \\
& 10,13 & 21,64 & 32,22 & 43,44 & 56,33 & 72,66 \\
\hline$[5,10]$ & $-0,39$ & 5,82 & 10,21 & 13,61 & 17,54 & 22,70 \\
& 17,87 & 21,71 & 31,12 & 42,88 & 56,36 & 72,62 \\
\hline$[5,50]$ & $-2,83$ & 1,02 & 5,31 & 10,32 & 18,21 & 22,90 \\
& 50,04 & 50,06 & 50,28 & 51,27 & 55,66 & 72,14 \\
\hline
\end{tabular}

Observa-se que o valor de amortecimento mínimo foi satisfeito para o sistema, exceto quando se requer um fator de $30 \%$ para o polo monitorado. Isso acontece porque o polo não monitorado $(-10,22 \pm j 14,22)$ tem seu fator de amortecimento reduzido de $58,36 \%$ (malha aberta) para $21,80 \%$ (quando ambos os ganhos iniciais são iguais a 5). A variação da posição dos polos durante o processo iterativo nessa situação é ilustrada na Fig. 7.

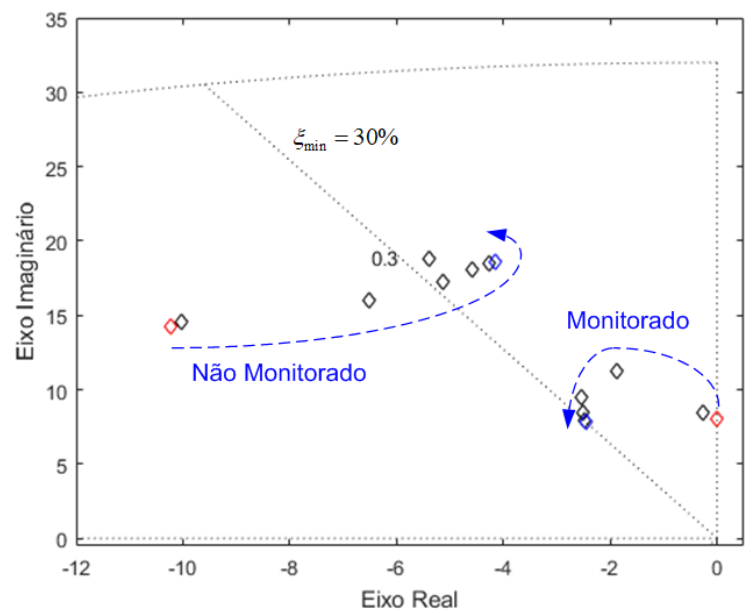

Fig. 7 Lugar das raízes para os dois modos eletromecânicos quando os ganhos são variados de zero até os valores ajustados $K_{I}=22,6$ e $K_{H}=$ 72,66

Com base nas simulações realizadas nota-se a importância da definição correta das condições iniciais dos ganhos e do número de polos monitorados. Quanto maior o número de polos, maior será o esforço computacional. Por outro lado, a redução da quantidade de polos considerados no processo pode implicar em amortecimentos pequenos e até mesmo negativos para polos não monitorados (caracterizando instabilidade em malha fechada).

Com o objetivo de validar a efetividade do controle projetado, a Fig. 8 apresenta a resposta do desvio de velocidade $\Delta \omega_{p u}$ a uma perturbação de 0,05 p.u. na tensão de referência $V_{R E F}$ do sistema de excitação (vide Fig. 3). É considerado o caso em que se desejou um fator de amortecimento igual a $15 \%$ partindo-se de ganhos iniciais iguais a 5: para 2 polos monitorados $\left(\mathrm{K}_{\mathrm{I}}=16,74 ; \mathrm{K}_{\mathrm{H}}=\right.$ $92,95)$ e 1 polo monitorado $\left(K_{I}=9,38 ; K_{H}=32,22\right)$. Notase a estabilização do sistema em ambas as situações.

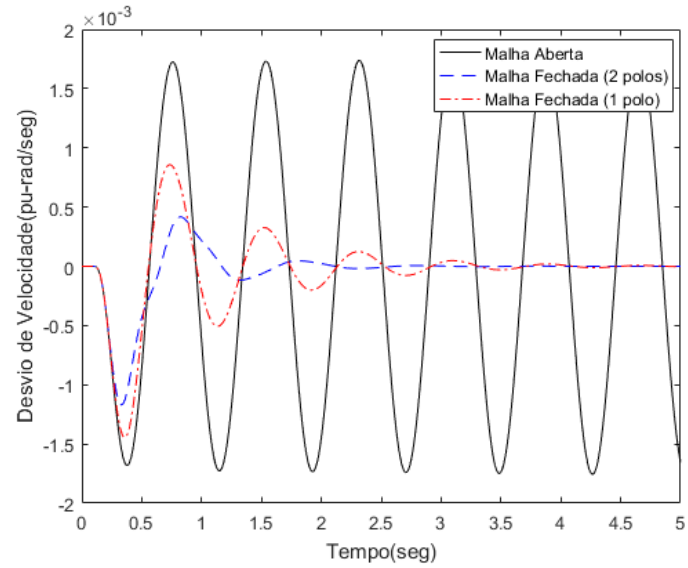

Fig. 8 Resposta no Domínio do Tempo.

Para os mesmos ganhos usados na resposta temporal, a Fig. 9 mostra a resposta em frequência dos controladores projetados considerando 1 ou 2 polos no processo de ajuste. Observa-se que a resposta em frequência é similar (as frequências centrais são iguais), diferindo-se no valor do ganho (maior quando se considera 2 polos no processo de ajuste).

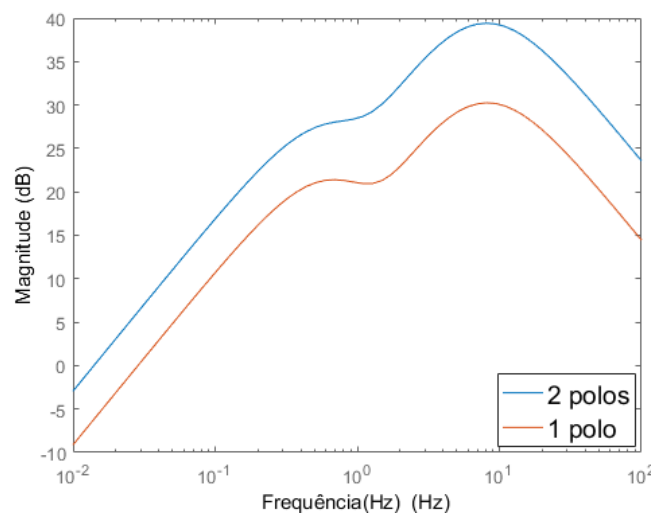

Fig. 9 Resposta em Frequência.

\section{CONCLUSÃO}

O presente trabalho apresentou uma metodologia para o ajuste de estabilizadores multi-banda via alocação de polos utilizando-se o método de Newton-Raphson. Objetiva-se alocar os polos em uma região adequada do plano complexo definida pelo mínimo fator de amortecimento. Embora se tenha considerado o sistema máquina barra infinita (pequeno porte), este foi adequado para a validação da metodologia proposta. As seguintes observações são destacadas:

a) a alocação de polos é parcial, isto é, seleciona-se um número de polos dominantes. Quanto maior o número de polos, mais oneroso será o custo computacional. Entretanto, a redução do número de polos monitorados no processo iterativo pode implicar em sistemas com baixo amortecimento e até mesmo instáveis (uma vez que o processo iterativo aloca somente os polos monitorados).

b) a seleção do número de polos é feita no início do processo iterativo e pode ser baseada na natureza dos polos (modos eletromecânicos de baixa frequência são prioridade). 
c) o método de Newton-Raphson, para cálculo de raízes de funções, é sensível às condições iniciais utilizada, o que foi observado na aplicação da metodologia proposta.

Como propostas de continuidade citam-se: (i) o ajuste coordenado de múltiplos ESP-MB e (ii) a consideração de múltiplos cenários operativos.

\section{AGRADECIMENTOS}

Os autores agradecem à FAPEMIG (projeto APQ02245-18), CAPES, CNPq e PPGEL/UFSJ pelo auxílio financeiro.

\section{REFERÊNCIAS}

Ferraz, J.C.R., Martins, N. and Taranto, G.N. (2007) 'Coordinated Stabilizer Tuning in Large Power Systems Considering Multiple Operating Conditions', 2007 IEEE Power Engineering Society General Meeting, 1-8.

Grondin, R., Kamwa, I., Trudel, G., Gerin-Lajoie, L. and Taborda, J. (2003) 'Modeling and closed-loop validation of a new PSS concept, the multi-band PSS', 2003 IEEE Power Engineering Society General Meeting, 1809 Vol. 3.

IEEE (2006) 'IEEE Std 421.5-2005 (Revision of IEEE Std 421.5-1992): 'IEEE recommended practice for excitation system models for power system stability studies".

Kamwa, I., Grondin, R. and Trudel, G. (2005) 'IEEE PSS2B versus PSS4B: the limits of performance of modern power system stabilizers', IEEE Transactions on Power Systems, vol. 20, May, pp. 903-915.

Peres, W., Silva Júnior, I.C. and Passos Filho, J.A. (2018) 'Gradient based hybrid metaheuristics for robust tuning of power system stabilizers', International Journal of Electrical Power \& Energy Systems, vol. 95, pp. 47-72.

Rimorov, D., Kamwa, I. and Joós, G. (2015) 'Model-based tuning approach for multi-band power system stabilisers PSS4B using an improved modal performance index', IET Generation, Transmission Distribution, vol. 9, pp. 21352143.

Sauer, P. and Pai, M.A. (1998) Power System Dynamics and Stability, Prentice Hall. 